(2) Open Access Full Text Article

\title{
Hepatotoxicity of tocilizumab and anakinra in rheumatoid arthritis: management decisions
}

\author{
Mahmud Mahamid 1,3 \\ Reuven Mader ${ }^{4}$ \\ Rifaat Safadi ${ }^{1,2}$ \\ 'Liver Unit, Holy Family Hospital, \\ Nazareth, Israel; ${ }^{2}$ Hadassah Medical \\ Center, Jerusalem, Israel; ${ }^{3}$ Shaare \\ Zedek Medical Center, Jerusalem, \\ Israel; ' ${ }^{4}$ heumatology Unit, Ha'emek \\ Medical Center, Afula, Israel
}

This article was published in the following Dove Press journal:

Clinical Pharmacology: Advances and Applications

4 October 2011

Number of times this article has been viewed
Background: Elevation of liver enzymes in rheumatoid arthritis patients treated with tocilizumab $\left(\right.$ Actemra $\left.^{\circledR}\right)$ or anakinra $\left(\right.$ Kineret $\left.^{\circledR}\right)$ is a well-documented phenomenon. However, characterization of liver histology has not been defined in most cases. Similarly, the factors involved in decisions regarding discontinuation of treatment and outcome have not been discussed in the literature to any significant extent.

Cases: Two women with rheumatoid arthritis refractory to standard therapies are reported here. One was treated with tocilizumab and the other with anakinra, and both developed toxic liver effects. Liver biopsy in both cases showed focal necrosis of hepatocytes - a hallmark of drug toxicity - with steatosis and early fibrosis. Inflammatory infiltrates were prominent in the patient treated with anakinra but not in the tocilizumab-treated patient. However, FibroTest (Assistance publique - Hôpitaux de Paris, Paris, France) in the latter patient showed an inflammatory activity of A2 and was staged as F2, and the histology also showed hemorrhagic areas. Although both patients were overweight and both had been exposed to steroids, the steatosis and steatohepatitis were considered to be related to drug hepatotoxicity. Other possible etiologies for liver injury were excluded. Discontinuation of anakinra led to rapid normalization of liver enzymes. The patient receiving tocilizumab developed hepatosplenomegaly but had normal liver enzymes. In spite of the hepatosplenomegaly, the tocilizumab treatment was continued since the patient had not responded to other drugs. There was a good response to the tocilizumab treatment and the liver biopsy showed only insignificant, reversible liver injury. At follow-up at 6-months the patient remains stable.

Conclusion: As cases showing tocilizumab or anakinra liver toxicity are appearing more frequently to the authors, a full assessment for liver injury is recommended in patients given those drugs, with careful consideration of the decision to continue or discontinue treatment. Further studies with long-term follow-up analysis are mandatory to guide appropriate management strategies.

Keywords: anakinra, interleukin receptors, liver injury, rheumatoid arthritis, tocilizumab

\section{Introduction}

Based on well-described efficacy and safety studies, tocilizumab (Actemra ${ }^{\circledR}$; Hoffman-La Roche, Basel, Switzerland) and anakinra (Kineret ${ }^{\mathbb{B}}$; Amgen Inc, Thousand Oaks, CA) have been approved for the treatment of rheumatoid arthritis in adults. ${ }^{1-3}$ Tocilizumab is a recombinant humanized anti-human interleukin-6 (IL-6) receptor monoclonal antibody and anakinra is an interleukin-1 (IL-1) receptor antagonist. ${ }^{2}$ Both drugs block inflammation and cartilage degradation associated with rheumatoid arthritis. ${ }^{4}$ Both tocilizumab and anakinra have been reported as being safe and well tolerated for up to
Correspondence: Mahmud Mahamid Digestive Diseases Institute, Shaare Zedek Medical Center, 12 Bayit Street, Jerusalem 91031 , Israel

Tel +97226666064

Fax +97226666050

Email mmahamid@szmc.org.il
(C) 201 I Mahamid et al, publisher and licensee Dove Medical Press Ltd. This is an Open Access article which permits unrestricted noncommercial use, provided the original work is properly cited. 
3 years of continuous use in a diverse population of patients with rheumatoid arthritis. ${ }^{1,2,5,6}$ They are given parenterally and may cause pain at the injection site. Other common reactions include headache, nausea, and flu-like symptoms. ${ }^{1,5}$ The most frequent serious adverse events are arthralgia and progression of the rheumatoid arthritis. ${ }^{1}$ Other adverse events may include infections, particularly pneumonia and upper respiratory infections, fractures, sinusitis, and diarrhea. Sepsis and opportunistic infection such as Mycobacterium or histoplasmosis are occasionally reported. ${ }^{1,2,5}$ In a large study of 1346 patients treated with tocilizumab or anakinra, a total of 15 fatalities occurred over 3 years of therapy. ${ }^{2}$ Events in the fatal cases included cardiac arrest, myocardial infarction, cerebrovascular accident, ventricular fibrillation, sepsis, upper gastrointestinal hemorrhage, malignant melanoma, lymphoma, and suicide. ${ }^{1,2,4}$

Liver injury in patients treated with both drugs is well recognized, and increased transaminases and serum bilirubin levels have been reported. ${ }^{1,2,5}$ In most cases of treatment with tocilizumab, the liver injury resolved or was alleviated following cessation or reduction in the dose of the drug. ${ }^{1-3,5}$ In general, neither the alaninaminotransferase elevations nor the increase in bilirubin were associated with clinical evidence of hepatic insufficiency. ${ }^{1}$ Indeed, in many cases there were other possible etiologies for the disturbance in liver function, such as autoimmune hepatitis, steroid-induced steatohepatitis, and even other drug-induced hepatotoxicities, particularly methotrexate (MTX). ${ }^{1,7}$ Although adverse effects of tocilizumab on the liver have been reported, histological evaluation of the liver pathology has not been documented.
The current report presents two patients with rheumatoid arthritis, with hepatotoxicity that may have been induced by tocilizumab and anakinra (Table 1).

\section{Case I}

A 46-year-old female who was first diagnosed as suffering from rheumatoid arthritis in May 2000. As nonsteroidal anti-inflammatory drugs (NSAIDs) were not effective, she was treated with MTX $12.5 \mathrm{mg}$ once weekly for 10 years. She developed progressive arthralgia and severe morning stiffness, and only high dosage systemic steroids succeeded in controlling the symptoms. MTX was discontinued, as it had ceased to become effective and did not achieve steroid sparing. In June 2009 she was treated with three courses of infliximab (Remicade ${ }^{\circledR}$; Janssen Biotech, Inc, Horsham, PA) against antitumor necrosis alpha (TNF $\alpha$ ). This was administered in a dose of $5 \mathrm{mg} / \mathrm{kg}$ (ie, $5 \mathrm{mg} \times 80 \mathrm{~kg}=400 \mathrm{mg}$ ) at weeks 0,2 , and 6 but did not result in clinical improvement. In January 2010, 6 months after the final course of infliximab, treatment was started with intravenous infusions of tocilizumab at a dose of $8 \mathrm{mg} / \mathrm{kg}$ (ie, $640 \mathrm{mg}$ ) - the maximal recommended dose. The patient received two infusions 1 month apart and then continued on a maintenance dose of $4 \mathrm{mg} / \mathrm{kg}$ (320 mg) monthly by intravenous infusion. She experienced a good response, with significant improvement in the arthralgia and morning stiffness.

Prior to tocilizumab administration, her total white blood cell count (measured in cells per cubic millimeter $[\mathrm{cmm}]$ ) was 7000/cmm (77\% neutrophils), hemoglobin $14 \mathrm{~g} / \mathrm{dL}$, and platelet count $178,000 / \mathrm{cmm}$. Full biochemical analy-

Table I Patient characteristics

\begin{tabular}{|c|c|c|}
\hline Baseline patient features & Case I & Case 2 \\
\hline Age & 46 & 49 \\
\hline Year of diagnosis & $2000 *$ & 1998* \\
\hline Sex & Female & Female \\
\hline Medical history & None & Infertility, hypertension, fatty liver \\
\hline Previous treatment for rheumatoid arthritis & NSAIDs, MTX, Remicade ${ }^{\circledR * *}$ & NSAIDs, MTX \\
\hline \multirow[t]{7}{*}{ Baseline laboratory tests } & $\mathrm{GOT}=22 \mathrm{U} / \mathrm{L}$ & $\mathrm{GOT}=16 \mathrm{U} / \mathrm{L}$ \\
\hline & $\mathrm{GPT}=18 \mathrm{U} / \mathrm{L}$ & $\mathrm{GPT}=16 \mathrm{U} / \mathrm{L}$ \\
\hline & $\mathrm{LDH}=250 \mathrm{U} / \mathrm{L}$ & $\mathrm{LDH}=364 \mathrm{U} / \mathrm{L}$ \\
\hline & $\mathrm{GGT}=25 \mathrm{U} / \mathrm{L}$ & $\mathrm{GG}=21 \mathrm{U} / \mathrm{L}$ \\
\hline & WBC (neutrophils \%) = $7000(77 \%)$ & WBC (Neutrophils \%) = 9,500 (60\%) \\
\hline & $\mathrm{HB}=14 \mathrm{~g} / \mathrm{dL}$ & $\mathrm{HB}=13.5 \mathrm{~g} / \mathrm{dL}$ \\
\hline & $\mathrm{PLT}=178,000 / \mathrm{cmm}$ & $\mathrm{PLT}=22 \mathrm{I}, 000 / \mathrm{cmm}$ \\
\hline Concomitant medications & None & Atenolol \\
\hline Body mass index & 25 & 31 \\
\hline Alcohol consumption & None & None \\
\hline History of liver disease & None & Nonalcoholic fatty liver disease \\
\hline
\end{tabular}

Notes: *American Rheumatism Association criteria ${ }^{13}$; **Remicade ${ }^{\circledR}$ (Janssen Biotech, Inc, Horsham, PA) against antitumor necrosis alpha (TNF $\alpha$ ).

Abbreviations: NSAIDs, nonsteroidal anti-inflammatory drugs; MTX, methotrexate; GOT, glutamic oxaloacetic transaminase; GPT, glutamic pyruvic transaminase; LDH, lactate dehydrase; GGT, gamma glutamyl transpeptidase; ALP, alkaline phosphatase; WBC, white blood cell; PLT, platelets; HB, hemoglobin. 
sis, including serum transaminases (glutamic oxaloacetic transaminase [GOT], glutamic pyruvic transaminase [GPT], lactate dehydrase $[\mathrm{LDH}]$, gamma glutamyl transpeptidase [GGT], and alkaline phosphatase [ALP]) were all within the normal ranges.

In February 2010, she started to complain of generalized weakness and fatigue. Laboratory tests at the time remained within normal limits. Abdominal ultrasound showed mild fatty liver as well as mild splenomegaly. Investigation of fatty liver disease and splenomegaly revealed negative (normal) results for serum hepatitis B surface antigen, anti-hepatitis $\mathrm{C}$ virus, human immunodeficiency virus, cytomegalovirus immunoglobulin M, Epstein-Barr virus immunoglobulin M, antinuclear antibodies, and anti-liver-kidney microsome antibodies. Serum ceruloplasmin, ferritin, thyroid-stimulating hormone, and glycated hemoglobin levels were also within normal limits. There was no history of other drug or herbal administration, no alcohol consumption, and no family history of liver disease.

On physical examination, the patient was found to be overweight; body mass index was 25 , which is mildly elevated. Apart from mild splenomegaly, the physical examination was unremarkable, with no other stigmata of chronic liver disease. Liver biopsy on July 2010 revealed focal hemorrhagic necrosis of hepatocytes with zones of macrovesicular steatosis and perisinusoidal fibrosis but no evidence of inflammation or cellular infiltrate (Figure 1). There was no evidence of hepatitis or any other cause of hepatotoxicity, and the histological findings of steatosis and focal necrosis were attributed to the tocilizumab. Fibro Test indicated injury activity of A1, and was also scored as a fibrosis index of F1. As the patient had failed to respond to all other therapeutic modalities it was decided to continue

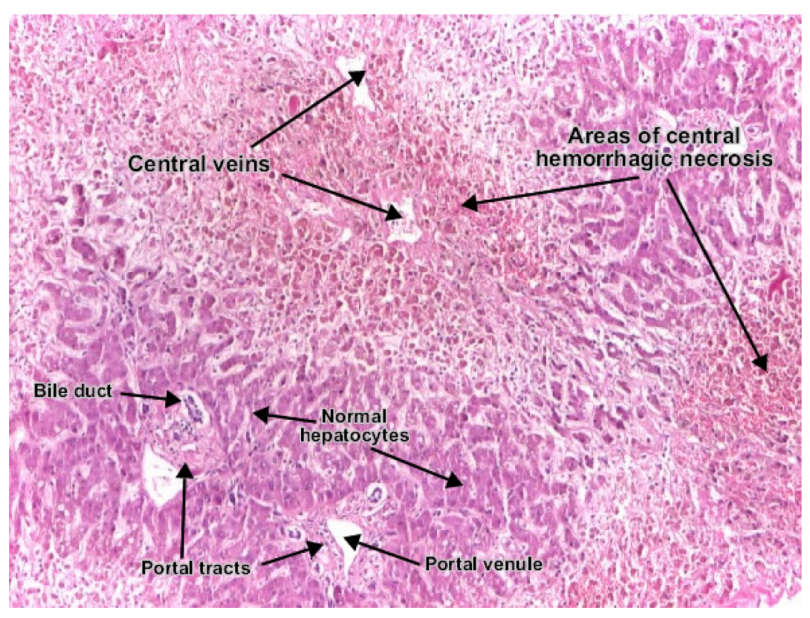

Figure I Case I: areas of hemorrhagic necrosis and steatosis. the tocilizumab. This decision was influenced by the normal hepatic enzyme levels and lack of severe liver injury. After 6 months of follow-up the patient was well, and repeated blood analysis remained normal. Her rheumatoid arthritis was monitored throughout on the basis of the American College of Rheumatology criteria.

\section{Case 2}

A 49-year-old female with a medical history of infertility, obesity (body mass index $=31$ ) and hypertension controlled by atenolol. The patient was also known to have nonalcoholic fatty liver disease (NAFLD) due to being overweight, confirmed by liver sonography, with normal liver function tests. She had received no treatment for the NAFLD. She had a 12-year history of rheumatoid arthritis, treated in the past with MTX and NSAIDs with only mild improvement. Complete blood count and biochemical data were all within the normal ranges (including GOT $16 \mathrm{U} / \mathrm{L}$, GPT $16 \mathrm{U} / \mathrm{L}, \mathrm{LDH} 364 \mathrm{U} / \mathrm{L}$, and GGT $21 \mathrm{U} / \mathrm{L}$ ).

Due to exacerbation of the signs and symptoms of rheumatoid arthritis, the patient was treated with $100 \mathrm{mg}$ /day anakinra by subcutaneous injection, starting in November 2009. In January 2010 she complained of fatigue and laboratory examination at that time revealed elevated levels of liver enzymes (GOT 30 U/L, GPT 50 U/L, LDH 584 U/L, ALP $161 \mathrm{U} / \mathrm{L}$, and GGT $85 \mathrm{U} / \mathrm{L}$; however, serum bilirubin and amylase levels were normal. There were no significant hematological changes (white blood cells, hemoglobin, and platelet counts). No coagulation abnormalities were detected and urinalysis was normal. Antinuclear antibody, anti-liver-kidney mircosome antibodies, serum hepatitis B surface antigen, anti-hepatitis $C$ virus, human immunodeficiency virus, immunoglobulin $\mathrm{M}$ for EpsteinBarr virus/cytomegalovirus, and anti-hepatitis A virus were all negative. Serum ferritin, ceruloplasmin, and serum thyroid-stimulating hormone levels were within normal limits. Physical examination showed an overweight woman with mild hepatosplenomegaly, but the remainder of the examination was unremarkable and there were no other stigmata of chronic liver disease. Repeated echogram of the upper abdomen showed fatty liver and hepatosplenomegaly. Liver biopsy in February 2010 showed macrovesicular steatosis in $80 \%$ of the biopsy volume, with focal necrosis of hepatocytes and mild inflammatory infiltrate of lymphocytes around the necrotic areas and the liver sinusoids (Figure 2). It further showed intraportal fibrosis and perisinusoidal fibrosis. On the basis of these findings, anakinra was discontinued after 2 months of administration. 


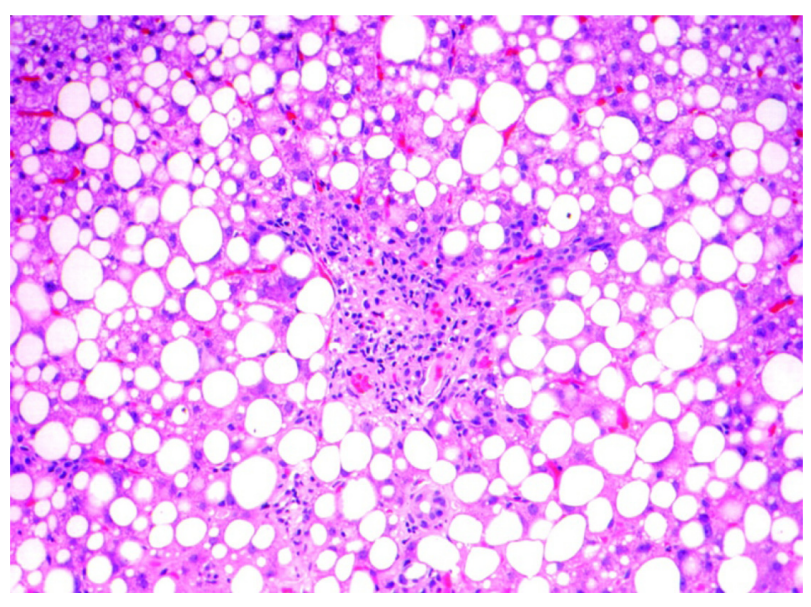

Figure 2 Case 2: macrovesicular steatosis in $80 \%$ of the biopsy volume with focal necrosis of hepatocytes and lymphocytic infiltrates.

She resumed treatment with NSAIDs and steroids, and refused any further treatment with biological drugs. After discontinuation of anakinra, her fatigue disappeared within 20 days, and the biochemical data improved gradually. Her rheumatoid arthritis was monitored throughout in accordance with the American College of Rheumatology criteria.

\section{Discussion}

This paper reports two women with active rheumatoid arthritis refractory to standard therapies. Due to ongoing complaints and progressive arthritis and arthralgia, associated with active inflammatory parameters, one woman was eventually treated with tocilizumab and the other with anakinra. Treatment with these drugs resulted in significant improvement in the rheumatoid signs and symptoms in both patients. As part of the routine protocol, hepatic side effects were carefully assessed. Blood tests and liver imaging indicated toxic liver effects in both women. Adverse hepatic effects of tocilizumab and anakinra are well described in rheumatoid arthritis patients. ${ }^{1-3,5,7}$ Similar to other reports in the literature, alanine transaminase elevation in these cases was not associated with clinically significant increases in direct bilirubin nor was it associated with clinical evidence of hepatic failure. ${ }^{1}$ To confirm that the hepatotoxicity was, indeed, drug related, an extensive search was undertaken for other causes of acute and chronic liver injury, and no such causes were found in either woman. This step is extremely important in such cases, since autoimmune hepatitis in association with rheumatoid arthritis is a well-known phenomenon. Moreover, NAFLD and nonalcoholic steatohepatitis were evident in the cases. This could have been related, at least in part, to the overweight and past exposure to prolonged steroid therapy in both our patients. The presence of splenomegaly in both cases can also extend the etiologic differential diagnosis and include Felty's syndrome, among others. ${ }^{1,4}$ Moreover, splenomegaly could be a predictor of cirrhosis, a reactive finding in longstanding immune disorders or as part of the metabolic syndrome. ${ }^{8}$

To completely exclude autoimmune hepatitis, evaluate drug toxicity, and assess the severity of liver injury, liver biopsy was performed in both cases. In both patients, the biopsy showed similar findings, consistent with drug injury. Those findings included focal necrosis of hepatocytes, with steatosis and early fibrosis. Inflammatory infiltrates were prominent in the anakinra-treated patient but not in the patient treated with tocilizumab. However, plasma cell infiltration was not seen and therefore autoimmune hepatitis could be excluded. In the absence of autoimmune hepatitis, focal necrosis of hepatocytes is very suggestive of drug toxicity; hepatocyte necrosis is also not common in NAFLD. Tocilizumab toxicity, however, is associated with hemorrhages but not with inflammatory infiltrates. FibroTest (Assistance publique - Hôpitaux de Paris, Paris, France) is a noninvasive tool recently developed to evaluate liver injury. This test was used in the tocilizumab-treated patient to assess liver injury and to serve as a noninvasive follow-up tool in the presence of normal levels of liver enzymes. It showed a necroinflammatory activity of A1-2 and was staged as F1-2. Thus, the findings of focal hepatocyte necrosis surrounded by either hemorrhages or inflammatory infiltrates in the patients were considered as direct histological evidence of drug toxicity related to the tocilizumab and anakinra. It has been suggested that the hepatic necrosis due to anakinra is a T-cell-dependent apoptotic immune-mediated response., ${ }^{910}$

The mechanism by which tocilizumab and anakinra are implicated in the pathogenesis of the fatty infiltrate formation is unclear. A role for the adipocytokines, leptin, plasminogen activator inhibitor-1, and insulin resistance has been offered to provide an etiopathological link. ${ }^{8,11,12}$ However, more studies are needed to confirm this complex mechanism in humans.

Discontinuation of anakinra in Case 2 led to a rapid normalization of liver enzymes. In the other case, treated with tocilizumab, there was only hepatosplenomegaly but with completely normal liver enzymes. Cessation or dosage reduction of tocilizumab has been reported to result in improvement in liver enzyme levels. In spite of the toxic effect demonstrated in Case 1, tocilizumab treatment was continued, in view of a lack of response to other drugs, the good response 
to tocilizumab, and the insignificant histological liver injury. After 6 months of follow-up the patient remains well.

\section{Conclusion}

As more and more patients are being treated with tocilizumab and anakinra who develop liver toxicity, it is recommended that a full assessment of liver injury take place and the decision to continue or discontinue the drug be carefully considered. More series with long-term follow-up are needed to develop a management strategy.

\section{Disclosure}

The authors report no conflicts of interest in this work.

\section{References}

1. Fleischmann RM, Tesser J, Schiff MH, et al. Safety of extended treatment with anakinra in patients with rheumatoid arthritis. Ann Rheum Dis. 2006; 65(8):1006-1012.

2. Donahue KE, Gartlehner G, Jonas DE, et al. Systemic review: comparative effectiveness and harms of disease-modifying medications for rheumatoid arthritis. Ann Intern Med. 2008;148(2):124-134.

3. Mertens M, Singh JA. Anakinra for rheumatoid arthritis. Cochrane Database Syst Rev. 2009;(1):CD005121.

4. Cunnane G, Madigan A, Murphy E, FitzGerald O, Bresnihan B. The effect of treatment with interleukin-1 receptor antagonist on the inflamed synovial membrane in rheumatoid arthritis. Rheumatology (Oxford). 2001;40(1):62-69.
5. Weisman MH. What are the risks of biological therapy in rheumatoid arthritis? An update on safety. J Rheumatol Suppl. 2002;65:33-38.

6. Garcea G, Maddern GJ. Liver failure after major hepatic resection. J Hepatobiliary Pancreat Surg. 2009;16(2):145-155.

7. Genovese MC, Cohen S, Moreland L, et al. Combination therapy with etanercept and anakinra in the treatment of patients with rheumatoid arthritis who have been treated unsuccessfully with methotrexate. Arthritis Rheum. 2004;50(5):1412-1419.

8. Juhan-Vague I, Alessi MC, Mavri A, Morange PE. Plasminogen activator inhibitor-1, inflammation, obesity, insulin resistance and vascular risk. J Thromb Haemost. 2003;1(7):1575-1579.

9. Mosmann TR, Moor KW. The role of IL-10 in crossregulation of TH1 and TH2 responses. Immunol Today. 1991;12(3):A49-A53.

10. Gewiese-Rabsch J, Drucker C, Malchow S, Scheller J, Rose-John S. Role of IL-6 trans-signaling in $\mathrm{CCl}_{4}$ induced liver damage. Biochim Biophys Acta. 2010;1802(11):1054-1061.

11. Lam SP, Luk JM, Man K, et al. Activation of interleukin-6-induced glycoprotein 130/signal transducer and activation of transcription 3 pathway in mesenchymal stem cells enhances hepatic differentiation, proliferation, and liver regeneration. Liver Transpl. 2010;16(10): 1195-1206.

12. Cressman DE, Greenbaum LE, DeAngelis RA, et al. Liver failure and defective hepatocyte regeneration in interleukin-6-deficient mice. Science. 1996;247(5291):1379-1383.

13. Arnett FC, Edworthy SM, Bloch DA, et al. The American Rheumatism Association 1987 revised criteria for the classification of rheumatoid arthritis. Arthritis Rheum 1988;31:315-324.
Clinical Pharmacology: Advances and Applications

\section{Publish your work in this journal}

Clinical Pharmacology: Advances and Applications is an international, peer-reviewed, open access journal publishing original research, reports, reviews and commentaries on all areas of drug experience in humans. The manuscript management system is completely online and includes a very quick and fair peer-review system, which is all easy to use

\section{Dovepress}

Visit http://www.dovepress.com/testimonials.php to read real quotes from published authors. 